\title{
High-frequency ultrasound in the diagnosis of selected non-melanoma skin nodular lesions
}

\author{
Paweł Piłat ${ }^{1,2}$, Adam Borzęcki², Mieczysław Jazienicki ${ }^{3}$, Agnieszka Gerkowicz' ${ }^{1}$, Dorota Krasowska ${ }^{1}$ \\ ${ }^{1}$ Chair and Department of Dermatology, Venerology and Pediatric Dermatology, Medical University of Lublin, Lublin, Poland \\ ${ }^{2}$ Non-Public Health Care Center Med-Laser, Lublin, Poland \\ ${ }^{3}$ Department of Surgical Oncology, $1^{\text {st }}$ Independent Public Clinical Hospital, Lublin, Poland
}

Adv Dermatol Allergol 2019; XXXVI (5): 572-580

DOI: https://doi.org/10.5114/ada.2019.89505

\begin{abstract}
Introduction: Ultrasonographic examination is commonly used in an outpatient setting, possibly due to its low cost, low risk for patients and the possibility to obtain real time images. Typically used heads have the frequency ranging from 7.5 to $12 \mathrm{MHz}$. Higher frequencies ensure higher resolution, yet they are limited by the penetration depth - reaching from several to several tens of millimetres into the skin. High-frequency ultrasonography (HFUS) appears to be a promising method for the detection and differential diagnostics of selected nodular skin lesions. Aim: The study aimed at a comparison of the data obtained by using HFUS, histopathological and dermatoscopic images of selected skin lesions to determine their common features.

Material and methods: Nodular lesions classified as potentially malignant were subjected to clinical, dermatoscopic and high-frequency ultrasonographic examinations. Then the patients were referred for surgical removal with histopathological assessment.

Results: A total of 54 nodular lesions were examined, out of which 34 were diagnosed as non-melanoma. The most common lesions were melanocytic naevi dermatofibroma, nodular basal cell carcinoma and pyogenic granuloma. Other examined lesions included blue naevus, seborrheic wart, xanthogranuloma juvenile and Spits naevus. In all lesions except Spitz naevus, HFUS images corresponded at least with dermatoscopic or histopathology images. Conclusions: HFUS can be used as a supporting diagnostic tool ensuring better pre-operative proceedings. HFUS is a non-invasive, easy and inexpensive screening method for the determination of different skin cancers as it provides valuable information allowing to determine the cutting margins and lesion shape.
\end{abstract}

Key words: blue nevus, seborrheic wart, xanthogranuloma juvenile, pyogenic granuloma, high-frequency ultrasonography, basal cell carcinoma.

\section{Introduction}

The basic tool in the daily work of a dermatologist, which is helpful in the diagnosis and differentiation of a single skin lesion is a dermatoscope. It allows the physician to perform a rapid evaluation of disease outbreaks and to make a good diagnosis. It is frequently necessary to evaluate the lesion holistically, including the thickness and sub- and intradermal extent, without histopathological examination of the skin continuity. This function is perfectly fulfilled by high-frequency ultrasonography (HFUS), enabling better diagnostics of selected nodular lesions. The studies published thus far in the field of skin cancers have proven that HFUS provides a large amount of valuable information [1-5].

\section{Aim}

The study aimed at imaging nodular lesions with the use of HFUS, initially qualified as potentially malignant and establishing the possible common features in the sonographic, dermatoscopic and histopathological images.

\section{Material and methods}

A total of 63 patients were qualified for the study in the period from April 2015 to December 2016, referred with suspected malignant lesions. Nine patients did not consent to continue the examination. The final group amounted to 54 patients, including 34 women and 20 men. The average age was $50.71 \pm 20$, the youngest

Address for correspondence: Paweł Piłat MD, Non-Public Health Care Center Med-Laser, 14 A Młyńska St, 20-406 Lublin, Poland, phone: +48 607760 179, e-mail: pawelpilat1@gmail.com Received: 8.05.2018, accepted: 14.08.2018. 
patient included in the group was 4 years of age, the oldest 88 years of age. All patients were provided with information and gave informed consent to participate in the examination. The Local Bioethics Committee issued positive opinion no. IWL/237/7123/2016 for the study. All patients were examined following the same scheme.

Initially each lesion was evaluated macroscopically, a photographic documentation was performed. Then, a dermatoscopic examination was performed using a manual dermatoscope DERMLITE ${ }^{\oplus}$ DL3 with $10 \times$ magnification and photographic documentation was performed.

Nodular lesions and healthy skin neighbouring disease-free tissues were examined using DermaScan C ultrasonography (Cortex Technology ApS) with the use of two ultrasonographic heads with a frequency of $20 \mathrm{MHz}$ (resolution from 60 to 200 microns and $23 \mathrm{~mm}$ penetration) and $50 \mathrm{MHz}$ (resolution of 60 microns and $3 \mathrm{~mm}$ penetration). The device DermaScan C is included on the FDA list as a medical product in regulatory class II/21 CFR 892,1560 (510 (k) no. (K983945). It ensures real time tissue scanning with a frequency of 6-8 frames per second.

Ultrasonographic examination was performed after application of standard sonography gel on the nodular lesion. Ultrasonography aimed at imaging of the sonographic structure and assessment of echogenicity in relation to the neighbouring disease-free tissues (hyper- or hypoechogenicity), measurement of the thickness of the imaged nodules from the distal part of the epidermis echo to the distal part of the lesion margin visible on the computer screen. In each patient, an ultrasonographic examination of healthy skin was performed.

All nodular lesions were surgically removed with a suitable margin, adequate to the lesion type. Subsequently, the removed tissues were subjected to histopathological examination.

\section{Results}

A total of 54 nodular lesions were examined, out of which 34 were diagnosed as non-melanoma skin lesions. In order to present them in the study, of those 34 we selected 17 cases for which there was little or no literature data available in the pubmed.gov. They had some characteristic features in the sonography examination (Table 1). The description of these lesions based on clinical, dermatoscopic, sonographic and histopathological images is presented in Table 2. The remaining 17 lesions turned out to be benign naevus on which there are many works presenting their high-frequency sonographic images. For this reason, we decided to omit their description in the present study.

\section{Discussion}

A quick and accurate recognition of a malignant lesion constitutes the most important objective of the modern dermatology. Thus, diagnostic methods enabling a precise, and the least invasive examination, are continuously searched. It appears that HFUS may be helpful in the process, enabling differentiation between malignant and benign lesions. The majority of already published works have concentrated on the use of HFUS in the diagnostics of melanoma and basal cell carcinoma [1-5]. Only few studies described the possible use of HFUS in the diagnosis of benign skin lesions [6-8]. In the present study, HFUS was used for imaging lesions initially identified as potentially malignant by experienced dermatologists. Thanks to the use of heads with a constant frequency, high resolution images were obtained, thus enabling delineation of their margins, dimensions and depth of the nodules, which translated into further therapeutic decisions. It is worthy of mention that almost 63\% of the lesions assessed after the second dermatoscopic examination, HFUS examination and histopathological

Table 1. Characteristics of the nodular lesions in the sonography examination

\begin{tabular}{|c|c|c|c|c|}
\hline Examined lesion & $\begin{array}{c}\text { Echogenicity } \\
\text { Hypo-/hyperechogenicity }\end{array}$ & Entry echo & Edges sharp/not sharp & $\begin{array}{l}\text { Lesion structure } \\
\text { e.g. uniform }\end{array}$ \\
\hline Healthy skin & Hyperechoic & Uniform & - & Uniform \\
\hline Nodular BCC & Hypoechoic & Not uniform & Sharp & Uniform \\
\hline Pyogenic granuloma & Hypoechoic & Uniform & Sharp & Uniform \\
\hline Dermatofibroma & Hypoechoic & Uniform & Not sharp & Uniform \\
\hline Spitz naevus & Hypoechoic & Not uniform & Sharp & Uniform \\
\hline Seborrheic keratosis & Hypoechoic & Not uniform & Sharp & Uniform \\
\hline Blue naevus & Hypoechoic & Not uniform & Not sharp & Not uniform \\
\hline Compound naevus & Hypoechoic & Not uniform & Sharp & Not uniform \\
\hline $\begin{array}{l}\text { Xanthogranuloma } \\
\text { juvenile }\end{array}$ & Hypoechoic & Uniform & Sharp & Uniform \\
\hline
\end{tabular}




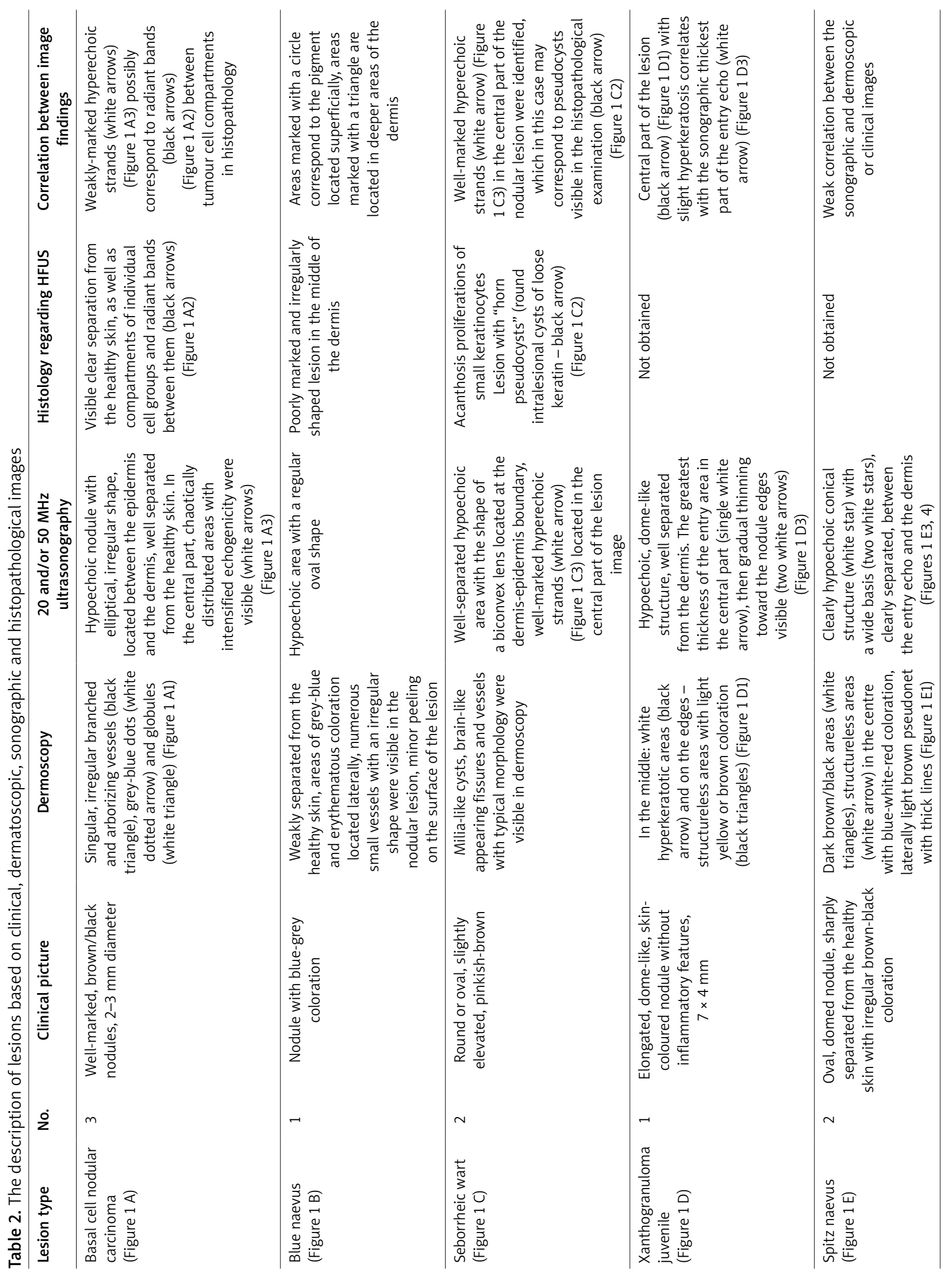




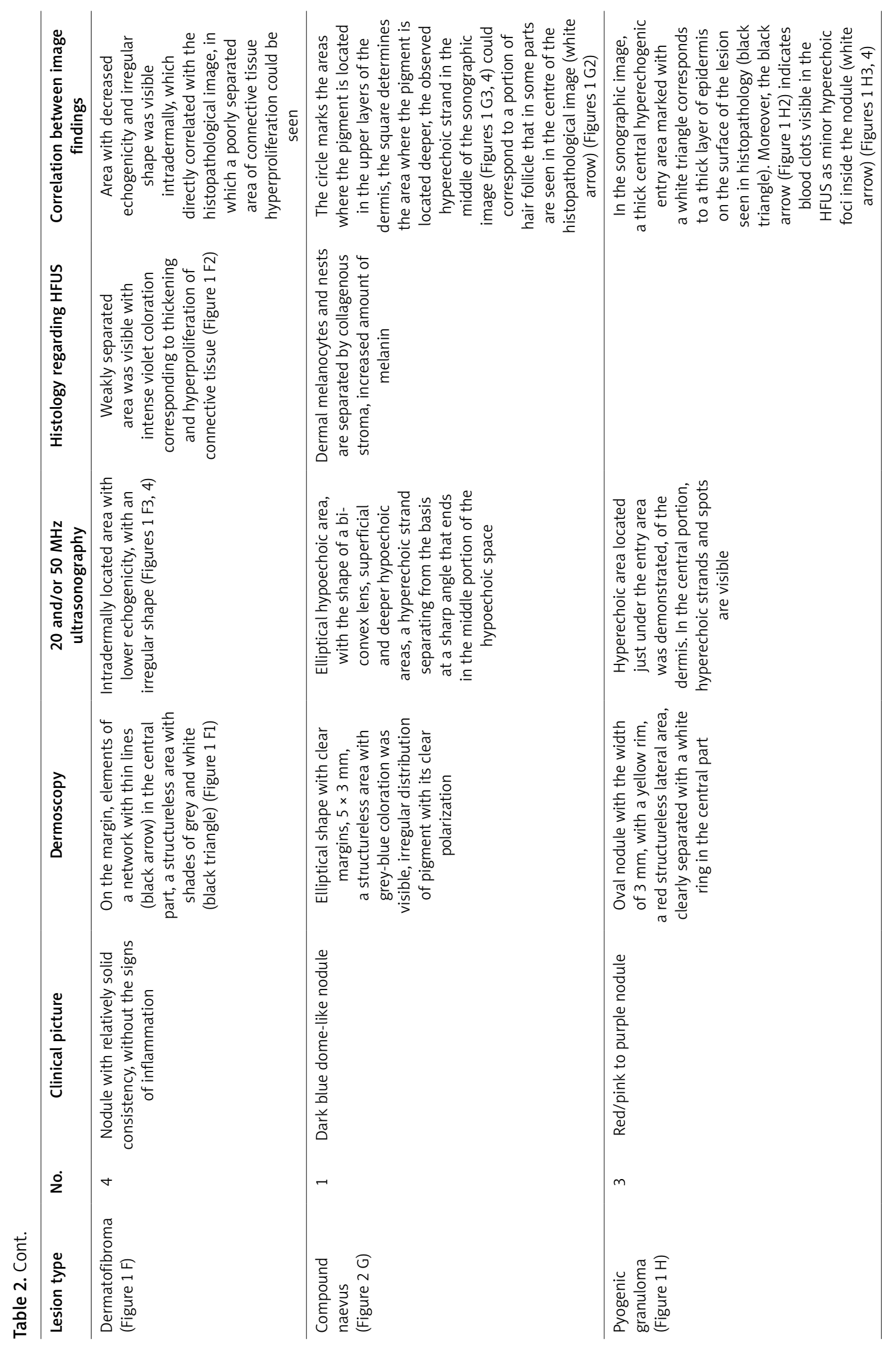



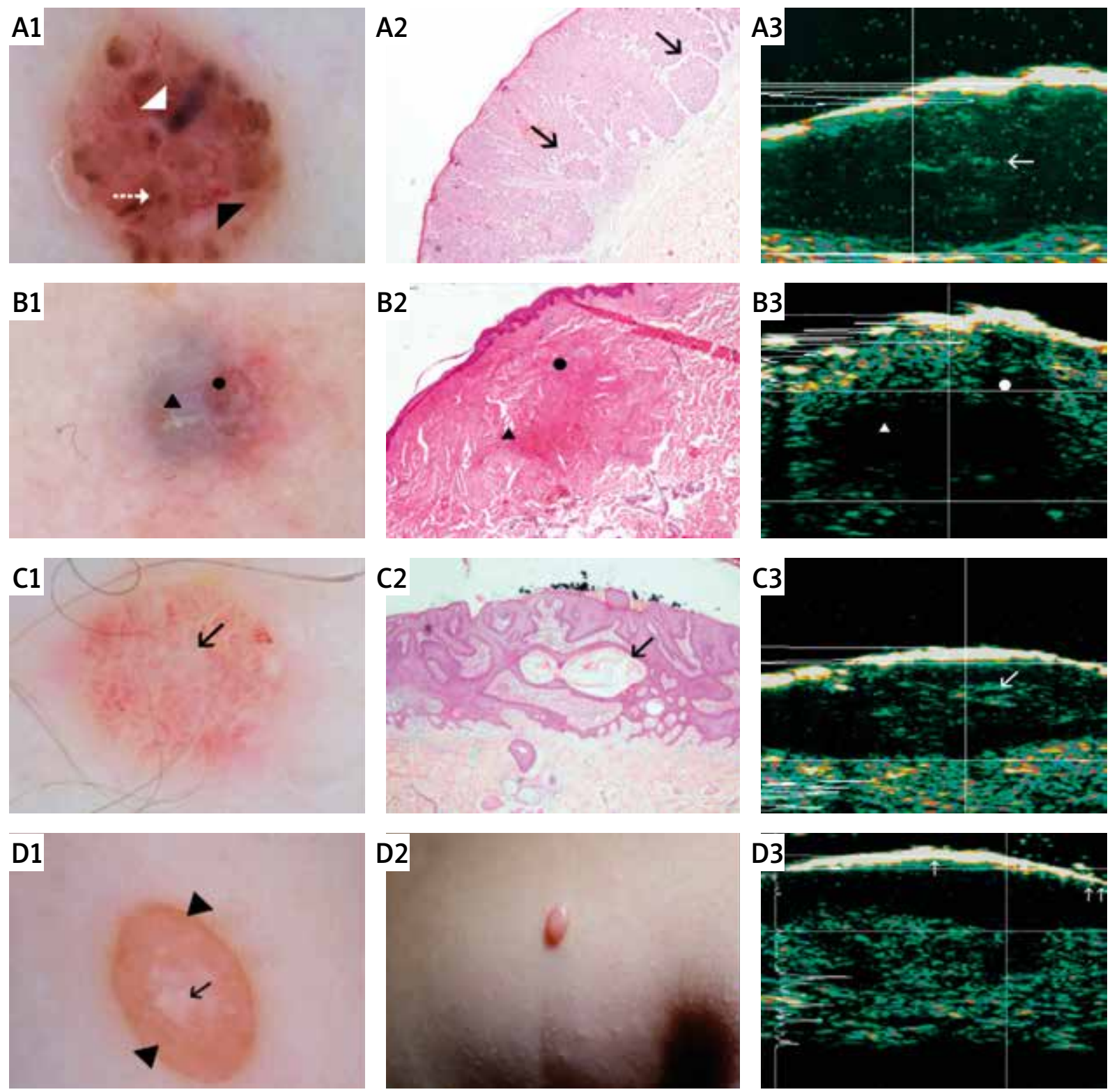

Figure 1. A - Basal cell nodular carcinoma: 1) dermoscopy: singular, irregular branched and arborizing vessels (black triangle), grey-blue dots (white dotted arrow) and globules (white triangle), 2) histopathology: compartments of individual cell groups and radiant bands between them (black arrows), 3) ultrasonography: chaotically distributed areas with intensified echogenicity (white arrows). B - Blue naevus: 1) dermoscopy, 2) histopathology, 3) ultrasonography. Pigment located superficial (black dot), pigment located in deeper areas of the dermis (black triangle). C - Seborrheic wart: 1) dermoscopy: milia-like cysts (black arrow), 2) histopathology: round intralesional cysts of loose keratin (black arrow), 3) ultrasonography: hyperechoic strands (white arrow). D - Xanthogranuloma juvenile: 1) dermoscopy: white hyperkeratotic areas (black arrow), structureless light yellow and brown areas (black triangles), 2) clinical image, 3) ultrasonography: the thickest part of the entry echo in the centre (single white arrow), thin entry echo on the edges (two white arrows)

confirmation turned out to be non-melanoma lesions. This may indicate the need to use different methods in the diagnostic process.

Basal cell carcinoma (BCC) is one of the most commonly occurring skin malignant tumours in humans, primarily on the face. Recent research has indicated an increased incidence of this tumour [9-11]. Despite the characteristic dermatoscopic image, nodular BCC may provide numerous diagnostic difficulties, both for novice and experienced dermatologists. In the present study, 3 nodular BCCs were examined. The image of hypoechoic structure with an irregular shape located between epidermis and the dermis obtained by us in the HFUS examination was similar to the BBC images described earlier in 

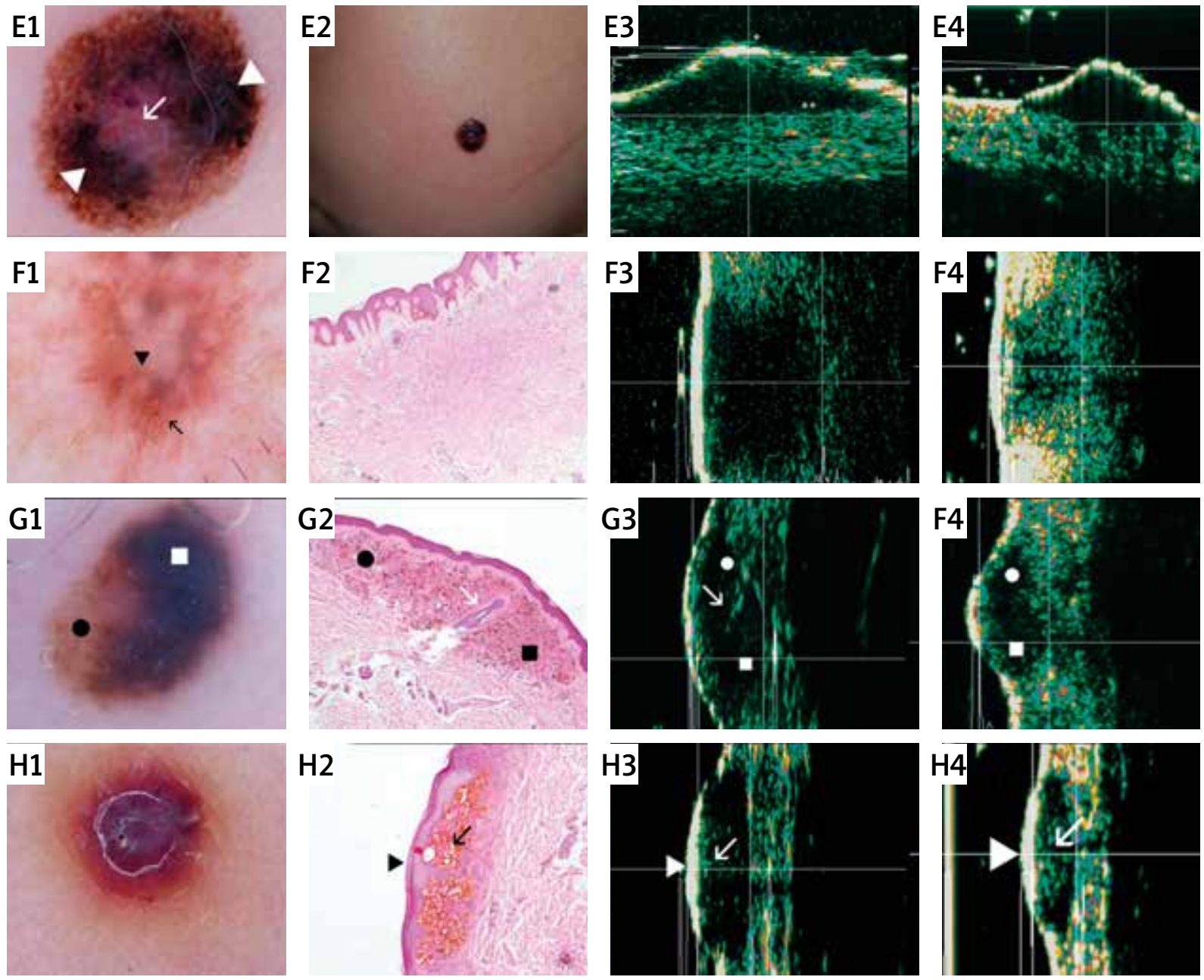

Figure 1. Cont. E - Spitz naevus: 1) dermoscopy: dark brown/black areas (white triangles), structureless blue/red/white areas (white arrow), 2) clinical image, 3) $50 \mathrm{MHz}$ ultrasonography: clearly hypoechoic conical structure (white star), wide basis (two white stars), 4) $20 \mathrm{MHz}$ ultrasonography. $\mathbf{F}$ - Dermatofibroma: 1) dermoscopy: network with thin lines (black arrow), shades of grey and white (black triangle), 2) histopathology: poorly defined proliferation of "fibrohistiocytic" cells within the dermis, 3) $50 \mathrm{MHz}$ ultrasonography: poorly marked intradermal hypoechogenic area, 4) $20 \mathrm{MHz}$ ultrasonography. G - Compound naevus: 1) dermoscopy, 2) histopathology, 3) $50 \mathrm{MHz}$ ultrasonography, 4) $20 \mathrm{MHz}$ ultrasonography. Pigment located in the upper layers of the dermis (black dot), pigment located in the deeper layer of the dermis (black square), parts of hair follicle (white arrow). $\mathbf{H}$ - Pyogenic granuloma: 1) dermoscopy, 2) histopathology: thick layer of epidermis on the surface of the lesion (black triangle), blood clots (black arrow), 3) $50 \mathrm{MHz}$ ultrasonography, 4) $20 \mathrm{MHz}$ ultrasonography: thick central hyperechogenic entry area (white triangle), blood clots visible in the HFUS as minor hyperechoic foci inside the nodule (white arrow)

HFUS examinations [6-8]. Furthermore, within the lesion we observed hyperechoic strands, which may correspond to connective tissue compartments between individual packets of cancerous cells visible in the histopathological examination. In the earlier study, Harland et al. suggested that the hyperechoic spots found within BCC may correspond to collagen bundles or keratin groups [12]. It is currently suggested to consider numerous hyperechoic spots within BCC as a characteristic feature enabling HFUS differentiation of BCC from melanoma, which does not contain such structures [13]. Considering a large percentage of recurrences, a precise pre-operative assessment of the lesion margins is of high importance in BCC therapy. Numerous publications emphasize the advantages of HFUS, considering it a method enabling a high precision assessment of lesion margins in the preoperation period, which translates to a selection of the appropriate cutting margins [14-16]. But, certain authors indicate the lack of usefulness of HFUS for pre-operative proceedings [17]. In the present study, the sonographic image of all BCC enabled surgical excision with the suitable margins.

Blue naevus often provides diagnostic difficulties due to its blue coloration, which is also characteristic of mel- 
anoma or its metastases, furthermore a dermatoscopic examination does not always allow for differentiation of these lesions [18-20]. Thus, the use of HFUS appears to be of particular interest in the diagnostics of blue naevi. There are only single studies describing sonographic images of blue naevi [7, 21]. Scotto di Santolo et al. reported the lack of the characteristic image of blue naevus in the HFUS examination. The authors observed both homogenous and non-homogenous nodules, with regular and irregular margins. On the other hand, vascularization was not found in any of the lesions in the sonographic examination using the colour Doppler method [7]. Our observations demonstrate that in the sonographic examination, blue naevus is shown as a hypoechoic, oval area, with the shape of an egg, located in the dermis, which allows to differentiate it from melanoma, which typically has the shape of a bi-convex lens in the sonographic examination. Of interest, sonographic images corresponded with dermoscopic and histopathological findings according to pigment location. This suggests the utility of HFUS in the diagnostic process. Moreover Samimi et al. demonstrated that HFUS with a frequency of $20 \mathrm{MHz}$ may be useful for the differentiation between blue naevus and melanoma metastases. The authors described melanoma metastases as hypoechoic, irregular areas (potato-shape), whereas blue naevi manifested as hypoechoic oval (egg-shape) areas [21].

A seborrheic wart is another common lesion, which may prove difficult in the differential diagnosis with skin malignant tumours. Dermatoscopic examination is the key diagnostic method here, yet in doubtful cases it is necessary to perform a cutting biopsy and histopathological verification [22-27]. Sonographic images using HFUS of a seborrheic wart have thus far been described in single publications only $[6,8,12]$. Typically, seborrheic warts produce the image of the hypoechogenic structure located in the epidermis with a thick entry echo, whereas the colour Doppler method does not determine the presence of vascularization $[6,8]$. In the present study we obtained a similar image, furthermore, within the lesion hyperechogenic structures that could correspond to milia-like cysts visible in the histopathological and dermatoscopic examination were observed. Although the obtained image of a seborrheic wart resembled a bi-convex lens, considered typical of a melanoma, the presence of hyperechogenic structures within the lesion appears to be the distinguishing feature from melanoma. Of particular interest is the study, which provided detailed differences in the sonographic image of melanoma and seborrheic warts [28]. These authors determined that the dermal echogenic ratio (DER) $>3$ is characteristic of the seborrheic wart and it allows for its differentiation from melanoma with $100 \%$ sensitivity and $79 \%$ specificity. Moreover, the latter value may be increased to 93\% when the presence of entry echo line enhancement (absent in melanomas) is considered [28].
Melanocytic lesions often require differentiation from melanoma. Apart from dermatoscopic examination, which is not always decisive, the possible use of HFUS has recently been emphasized, particularly for the monitoring of birthmarks and dysplastic naevi [6, 12]. Melanocytic lesions typically produce an image of round or oval hypoechogenic structures with clear margins. The presence of sharp margins is considered to be the distinguishing feature from melanoma [8]. In the present study, one compound naevus was examined. After comparison between dermatoscopic, histological and HFUS images, pigment distribution was presented at different depths in all obtained images. The possibility to determine the lesion depth, monitoring of its structures and regression areas with the use of HFUS is particularly important, for a cancerous transformation when the growth inside begins [6].

Dermatofibromas are benign lesions, which can be located on the skin of the entire body, but are particularly common on the lower (41\%) and upper (24\%) limbs. Numerous histological forms of dermatofibromas exist, which can produce various images in the dermatoscopic examination $[29,30]$. Thus, in doubtful cases a HFUS examination is recommended [31]. In the present study, 4 dermatofibromas were examined, obtaining hypoechogenic structures with an irregular shape. Furthermore, the obtained HFUS images corresponded with an irregular area of increased density of the connective tissue observed in the histopathological examination. Similar sonographic images were observed by other authors as a characteristic feature assuming weakly separated oval hypoechogenic nodule [31, 32]. But, a group of Italian researchers described 2 dermatofibromas, which in the HFUS examination had a non-homogenous echostructure with regular margins, as well as a lack of vascularization determined in a colour-Doppler examination [7].

Xanthogranuloma juvenile was another interesting lesion we examined. This rare form of histiocytosis is characterized by a benign course and the nodules may be located on the head, neck, body, limbs and genital organs. The involvement of the internal organs often takes place [33-35]. In the sonographic image we obtained xanthogranuloma juvenile manifested as a hypoechoic area located on the dermal-epidermal boundary. Furthermore, a clear enhanced entry echo (EEE) and clear posterior attenuation (PA) were visible. Of interest the thickest part of EEE corresponded with middle white areas visible during dermoscopy. A similar image was also observed in a 7-year-old girl [36]. Worthy of emphasis is the fact that the image we obtained with the use of HFUS differed from the image observed in the colour Doppler ultrasonography, which demonstrated a well-separated hypoechogenic nodule, yet the posterior attenuation was not observed [37]. Unfortunately, we do not have the histopathological image to which we could refer. 
In the present study, 2 Spitz naevi were imaged, including one in a 4-year-old female patient. According to our knowledge, there are no studies describing the use of HFUS in such small children. The image we obtained did not differ from the lesions described for the remaining age groups [7].

We also examined 3 pyogenic granulomas using HFUS. According to our knowledge, no studies have been published describing the possible use of HFUS in this disease. Colour Doppler ultrasonography was used in the studies published thus far. Despite the different diagnostic methods, the sonographic image described by other authors [38] was similar to that observed in the present study. It is worth mentioning that both sonographic and histopathological images obtained in the study exhibited many common features. The visible EEE in the sonographic image perfectly corresponded to the thicker layer of epidermis above the lesion in comparison to healthy skin. Furthermore, numerous hyperechoic areas corresponded to minor clots equally distributed in the central portion of the lesion.

The study presents clinical cases which were referred to as potentially cancerous lesions. In order to perform the evaluation, a clinical, dermatoscopic, ultrasonographic and histopathological examination was performed. The possible relations between dermatoscopic, ultrasonographic and histopathological images were evaluated. The above study was limited by a brief timeframe and a low number of patients qualified for the examination. This directly translated to a low number of cases described. The study is being continued and the obtained results will be published soon.

\section{Conclusions}

The presented preliminary results indicate that HFUS combined with dermatoscopy may be a helpful tool in the differential diagnostics of non-melanoma skin lesions. HFUS allows for better preparation for dermatologic procedures and often allows for the selection of suitable cutting margins.

Furthermore, an examination using HFUS is relatively quick, does not prolong the visit time and is not linked to negative feelings of the patient. It is repeatable and easy to perform and it may provide valuable information in doubtful cases. But, due to the low number of studies on the discussed topic it is necessary to conduct further research aiming at the development of diagnostic criteria facilitating HFUS examination and allowing for result standardization.

\section{Conflict of interest}

The authors declare no conflict of interest.

\section{References}

1. Bobadilla F, Wortsman X, Munoz C, et al. Pre-surgical high resolution ultrasound of facial basal cell carcinoma: correlation with histology. Cancer Imaging 2008; 8: 163-72.

2. Hayashi K, Koga H, Uhara H, Saida T. High-frequency 30-MHz sonography in preoperative assessment of tumor thickness of primary melanoma: usefulness in determination of surgical margin and indication for sentinel lymph node biopsy. Int J Clin Oncol 2009; 14: 426-30.

3. Vilana R, Puig S, Sanchez M, et al. Preoperative assessment of cutaneous melanoma thickness using 10-MHz sonography. AJR Am J Roentgenol 2009; 193: 639-43.

4. Gambichler T, Moussa G, Bahrenberg K, et al. Preoperative ultrasonic assessment of thin melanocytic skin lesions using a 100-MHz ultrasound transducer: a comparative study. Dermatol Surg 2007; 33: 818-24.

5. Guitera P, Li LX, Crotty K, et al. Melanoma histological Breslow thickness predicted by $75-\mathrm{MHz}$ ultrasonography. Br J Dermatol 2008; 159: 364-69.

6. Mandava A, Ravuri PR, Konathan R. High-resolution ultrasound imaging of cutaneous lesions. Indian J Radiol Imaging 2013, 23: 269-77.

7. Scotto di Santolo M, Sagnelli M, Mancini M, et al. Highresolution color-Doppler ultrasound for the study of skin growths. Arch Dermatol Res 2015; 307: 559-66.

8. Bhatt KD, Tambe SA, Jerajani HR, Dhurat RS. Utility of highfrequency ultrasonography in the diagnosis of benign and malignant skin tumors. Indian I Dermatol Venereol Leprol 2017; 83: 162-82.

9. Rubió-Casadevall J, Hernandez-Pujol AM, Ferreira-Santos MC, et al. Trends in incidence and survival analysis in non-melanoma skin cancer from 1994 to 2012 in Gerona, Spain: a population-based study. Cancer Epidemiol 2016; 45: 6-10.

10. Callens J, Van Eycken L, Henau K, Garmyn M. Epidemiology of basal and squamous cell carcinoma in Belgium: the need for a uniform and compulsory registration. J Eur Acad Dermatol Venereol 2016; 30: 1912-8.

11. Lomas A, Leonardi-Bee J, Bath-Hextall F. A systemic review of worldwide incidence of non melanoma skin cancer. Br J Dermatol 2012; 166: 1069-80.

12. Harland CC, Bamber JC, Gusterson BA, Mortimer PS. High frequency, high resolution B-scan ultrasound in the assessment of skin tumours. Br J Dermatol 1993; 128: 525-32.

13. Uhara H, Hayashi K, Koga H, Saida T. Multiple hypersonographic spots in basal cell carcinoma. Dermatol Surg 2007; 33: 1215-9.

14. Gupta AK, Turnbull DH, Foster FS, et al. High frequency 40$\mathrm{MHz}$ ultrasound. A possible noninvasive method for the assessment of the boundary of basal cell carcinomas. Dermatol Surg 1996; 22: 131-6.

15. Desai TD, Desai AD, Horowitz DC, et al. The use of high-frequency ultrasound in the evaluation of superficial and nodular basal cell carcinomas. Dermatol Surg 2007; 33: 1220-7.

16. Nassiri-Kashani M, Sadr B, Fanian F, et al. Preoperative assessment of basal cell carcinoma dimensions using high frequency ultrasonography and its correlation with histopathology. Skin Res Technol 2013; 19: 132-8.

17. Jambusaria-Pahlajani A, Schmults CD, Miller CJ, et al. Test characteristics of high-resolution ultrasound in the preoperative assessment of margins of basal cell and squamous cell carcinoma in patients undergoing Mohs micrographic surgery. Dermatol Surg 2009; 35: 9-15. 
18. Sakamoto S, Oiso N, Narita T, Kawada A. Blue nevus with a dermatoscopic appearance of peripheral streaks with branches. Case Rep Dermatol 2014; 6: 66-8.

19. Shiga T, Nakajima K, Tarutani M, et al. Blue nevus with a starburst pattern on dermoscopy. Dermatol Pract Concept 2012; 2: 204-7.

20. Sahin MT, Demir MA, Yoleri L, et al. Blue naevus with satellitosis mimicking malignant melanoma. J Eur Acad Dermatol Venereol 2001; 15: 570-3.

21. Samimi M, Perrinaud A, Naouri M, et al. High-resolution ultrasonography assists the differential diagnosis of blue naevi and cutaneous metastases of melanoma. Br J Dermatol 2010; 163: 550-6.

22. Yagerman SE, Marghoob AA. Clonal seborrheic keratosis ver sus epidermal nevus. J Am Acad Dermatol 2013; 69: 43-4.

23. Salerni G, Alonso C, Gorosito M, Fernández-Bussy R. Seborrheic keratosis-like melanoma. J Am Acad Dermatol 2015; 72: 53-5.

24. Ramyead S, Diaz-Cano SJ, Pozo-Garcia L. Dermoscopy of clonal seborrheic keratosis. J Am Acad Dermatol 2015; 73: 47-9.

25. Minagawa A, Tanaka M, Koga H, Okuyama R. Pigmented seborrheic keratosis showing starburst pattern. J Am Acad Dermatol 2016; 75: 11-3.

26. Repertinger S, Wang J, Adickes E, Sarma DP. Melanoma insitu arising in seborrheic keratosis: a case report. Cases J 2008; 1: 263.

27. Santos BS, Ribeiro LH, Ayres EL, Vilar EA. Comedo-like openings in melanoma. An Bras Dermatol 2014; 89: 344-6.

28. Harland CC, Kale SG, Jackson P, et al. Differentiation of common benign pigmented skin lesions from melanoma by high-resolution ultrasound. Br J Dermatol 2000; 143: 281-9.

29. Şenel E, Yuyucu Karabulut Y, Doğruer Şenel S. Clinical, histopathological, dermatoscopic and digital microscopic features of dermatofibroma: a retrospective analysis of 200 lesions. J Eur Acad Dermatol Venereol 2015; 29: 1958-66.

30. Zaballos P, Puig S, Llambrich A, Malvehy J. Dermoscopy of dermatofibromas: a prospective morphological study of 412 cases. Arch Dermatol 2008; 144: 75-83.

31. Zarchi K, Kromann CB, Wortsman X, Jemec GB. Usefulness of ultrasound for the diagnosis of dermatofibroma. Med UItrason 2016; 18: 132-3.

32. Wortsman X, Wortsman J. Clinical usefulness of variablefrequency ultrasound in localized lesions of the skin. J Am Acad Dermatol 2010; 62: 247-56f.

33. Pajaziti L, Hapçiu SR, Pajaziti A. Juvenile xanthogranuloma: a case report and review of the literature. BMC Res Notes 2014; 7: 174

34. Bradford RK, Choudhary AK. Imaging findings of juvenile xanthogranuloma of the penis. Pediatr Radiol 2009; 39: 176-9.

35. Szczerkowska-Dobosz A, Kozicka D, Purzycka-Bohdan D, et al. Juvenile xanthogranuloma: a rare benign histiocytic disorder. Adv Dermatol Allergol 2014; 31: 197-200.

36. Martínez-Morána C, Echeverría-Garcíaa B, Tardíob JC, Borbujoa J. Ultrasound appearance of juvenile xanthogranuloma imagen ecográfica de xantogranuloma juvenile. Actas Dermosifiliogr 2017; 108: 683-5.

37. Niklitschek S, Niklitschek I, González S, Wortsman X. Color Doppler sonography of cutaneous juvenile xanthogranuloma with clinical and histologic correlations. J Ultrasound Med 2016; 35: 212-3.

38. Cantisani V, Del Vecchio A, Fioravanti E, et al. Color-Doppler US features of a pyogenic granuloma of the upper dorsum tongue. J Ultrasound 2016; 19: 67-70. 